\author{
International Journal of Innovative Research in \\ Electrical, Electronics, Instrumentation and Control Engineering
}

Vol. 6, Issue 11, November 2018

\title{
Energy and QoS Efficient Algorithm for Wireless Body Area Networks
}

\author{
Mr. Niraj Korde ${ }^{1}$, Prof. Urmila Deshmukh ${ }^{2}$ \\ Department of Electronics \& Telecommunication, Indira College of Engineering and Management, \\ SPPU (University of Pune) $)^{1,2}$
}

\begin{abstract}
Due to emergence of WBAN in real time applications recently, significant research works are reported on communication standards of wireless body area networks (WBANs). The two primary challenges in opportunistic WBANs that are- network management cost reduction and energy consumption reduction in order to deliver the costeffective and reliable services to critical patients in healthcare application. There are number of solutions reported to address this challenges in recent past, however, they failed to cover all the aspects of WBANs communication. In this, project we proposed novel opportunistic communication protocol for WBANs with aim to solve the research challenges not only the energy efficiency and network management cost reduction but also solves the problem of non-reliable nodes data dissemination. First we proposed the novel energy-efficient and distributed network management cost minimization framework for dynamic connectivity and data dissemination in opportunistic WBANs. Then we proposed a pricing based approach for reliable node data dissemination. This can be done by reputation-based incentive methods to motivate participants to disseminate reliable data in participatory sensing system, while minimizing incentive cost for maintaining sufficient number of reliable participants. The simulation results reveal the effectiveness of proposed method.
\end{abstract}

Keywords: Cost Management, Energy Management, Opportunistic Communications, Reliable Data Dissemination, Wireless Body Area Networks (WBAN)

\section{INTRODUCTION}

Wireless Body Area Networks (WBAN)/Wireless Body Area Sensor Network (WBASN) consist of sensor nodes attached in and around the human body to monitor the bio signals of human being for a variety of applications such as patient monitoring, gaming etc. This term has been first coined by Van Dam et al (2001) and received the interest of several researchers. Due to the advancements in MEMS and wireless communication technologies, WBAN has undergone a technical boom in the last decade. The schematic overview of differences between Wireless Sensor Networks and Wireless Body Area Networks is given by [1]. There are significant points to be noted in the Wireless body area network. As opposed to the wireless sensor network, the WBAN monitoring environment is restricted to the human body, heterogeneous data rate, the requirement of biocompatible sensor devices and more variable network topology due to body movement.

The communication in the Body sensor network is categorized into two types, in body communication is the RF communication between invasive sensor nodes implanted inside the human body and on body communication is the communication between wearable sensor nodes. The MICS (Medical Implantable Communication Service) band - 402$405 \mathrm{MHz}$ should be used for in body communication (Sana Ullah et al 2010a). ISM or UWB can be used for on body communication.

A WBAN provides real-time electronic healthcare services to medically emergent patients in a cost effective manner. In a WBAN, several body sensor nodes are implanted on/in the human body to sense the physiological signals of patients. After sensing the physiological signals, the sensor nodes send the sensed data to the Local processing Unit (LPU). Subsequently, the LPU transmits the aggregated data to the local access points (APs), which, in turn, send them to the medical servers [3], [4]. The body sensor nodes transmit the medical data to LPUs at wide range of data rates from 10 $\mathrm{Kb} / \mathrm{s}$ to $10 \mathrm{Mb} / \mathrm{s}$ [5]. Also, the energy consumption rates of sensor nodes are restricted to certain limits, as the battery power of these nodes is limited. To minimize energy consumption, the sensor nodes use a one-hop star topology to send their medical data [6]. However, mobility, body postures, and environmental obstacles increase the dynamism in WBANs, which frequently changes the network topology, which, in turn, decreases the network QoS. Additionally, the link-quality between nodes in WBANs varies as a function of time due to various body movements, which also affects the inter-node connectivity [7].

Due to body movements and mobility of WBANs [6], the link qualities of intra-BAN and inter-BAN communication units degrade significantly, which increases the packet loss rate and decreases the life-time of the body sensor nodes. 


\section{International Journal of Innovative Research in Electrical, Electronics, Instrumentation and Control Engineering}

Vol. 6, Issue 11, November 2018

Further, the above also disrupts data dissemination. Therefore the QoS management cost in the network increases in order to maintain fair QoS among WBANs. As the network connectivity establishment and QoS management costs increase in the network, we need a network management cost minimization framework to provide reliable and cost effective service to WBANs. We introduced energy efficient and QoS efficient communication protocol for WBAN based on energy management cost minimization and QoS management cost minimization with reliable data dissemination.

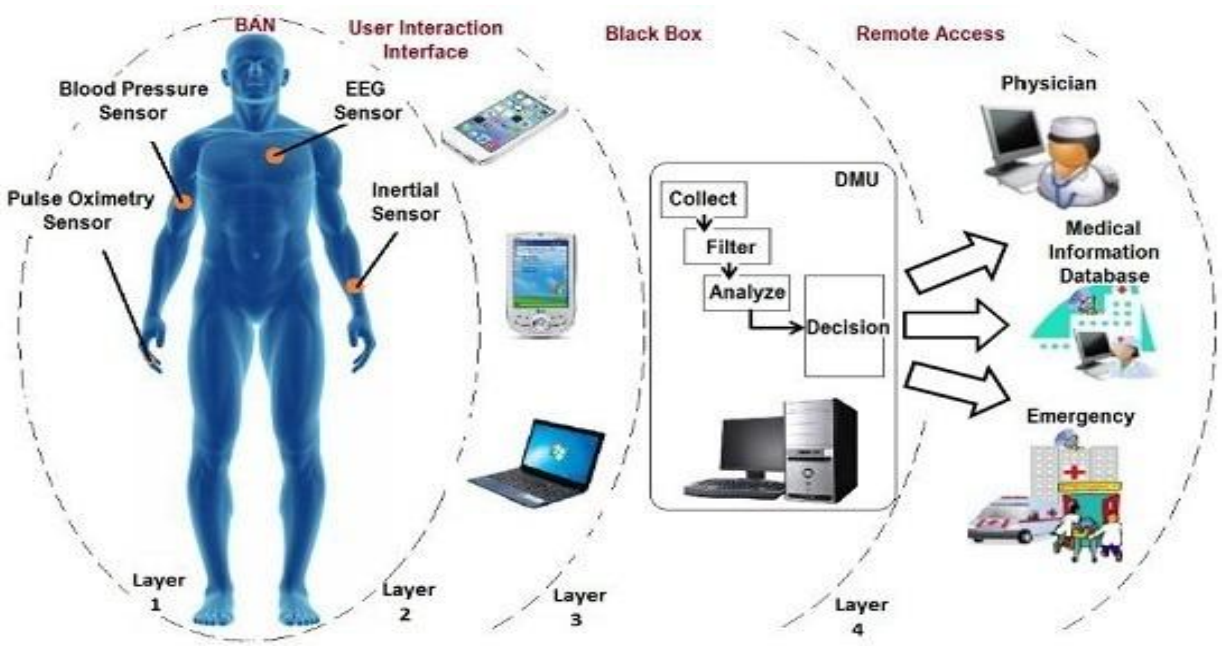

Figure 1: WBANs working scheme

\section{RELATED WORKS}

In this section, we represent the review of research works on practical communication protocol and energy consumption techniques for WBANs.

Samanta et al. [8], [9] investigated that link-quality-mindful asset assignment cum load adjusting plan for the hub in WBANs. In this system, the researcher has used two sub-issues dynamic resource portion and link-quality measurement in WBANs. These research works manage the adaptability of WBANs they don't examination the progression of network organization cost within the sight of body/ limb action in WBANs, in light of which the aggregate whole of network cost raising and the QoS of WBANs.

Elias at al. [10] innovated that an energy mindful ideal plan of energy successful and furthermore, financially savvy WBANs. This framework analyzed a financially savvy way; the analyst does not inspect the outcomes of dynamically exists and opportunistic information diffusing in WBANs. This raising the deferment and packet killed of the network.

Zhao et al. [11] investigated a network price minimization scheme for information fragmentation in WSNs. In the first place, this procedure does not inspect the required record of WBANs in the best way to deal with the survey the therapeutic state of the WBAN- devices patients, which is one of the extraordinary qualities of WBAN-based Deliberation.

Energy-effective and dependable Deliberation are essential requests of WBANs; these are supplies touchy therapeutic data because of shadowing and disappear outcomes inside the network, the energy utilization rate of sensor focus points increments, what's more, the trustworthiness in bits of knowledge transmission lessons, intermittently. To explosion the power execution and immovable quality in bits of knowledge transmission recently wide variety of strategies reported.

Yousaf et al. [12] investigated that, another three-level helpful transferring plan for WBANs. As casing sensor hubs deliver clinical insights at a variable charge, their relating activity design is unverifiable in nature. Subsequently, within the sight of negative hyperlink-agreeable, the parcels drop the charge the network and the power confirmation of body sensor focus point expands.

Andreagiovanni and Nardin [13] analyzed that vigorous approach for combine streamlining of energy correctness and information rate in WBANs under development absence of value. In this advancement work, the bundle transmission rate of body sensor center is thought to be homogenous in nature; regardless, in the province of WBANs, the packet transmission rate of body sensor focus points is heterogeneous.

Huang and Cai [14] researched proposed a concern conscious booking plan for utilized by a WBAN inside the presence of more than one coinciding WBANs. To grow the group throughput of WBANs, a nonlinear development trouble is portrayed, while thinking about the demands between WBANs. This work is obliged to handiest the stress mindful booking of WBANs inside the closeness of impedance between matching WBANs. Notwithstanding, they do never again think about the elevated network association value inside seeing impedance. 


\section{International Journal of Innovative Research in Electrical, Electronics, Instrumentation and Control Engineering}

Vol. 6, Issue 11, November 2018

Ibarra et al. [15] investigated a combined power and QoS control structure to plans of energy and got the best QoS in WBANs. The base paper techniques are analysis the difficulty traffic ministry for the information transference system. Be that as it may, this method isn't utilized for a wide range of traffic administration in the network.

Similarly, Seyedi at al. [16] evolved one-of-a-kind energy efficient data transmission scheme for WBANs. As a way to provide power-green statistics transmission, the tradeoffs among energy admission and packet mistakes possibility are implanted into the sensor center points. This frame neglects to offer QoS to WBANs inside sight of community dynamics, on due of flexibility.

Also, Seyedi et al. [17] researched that a Markov-chain depend analytical approach for energy aggregate hub in WBANs, in which the probability of circumstance hardship is examined to see the energy hardship circumstance of the body sensors center point. Ren et al. [18] explored an approach which secures higher network throughput for synchronize WBANs. The base paper works does not fulfil to supplies robustness in the network management approach for WBANs.

\section{PROPOSED DESIGN}

To solve the current research problem for dynamic WBAN communications, we proposed novel opportunistic communication protocol for WBANs with aim to solve the research challenges not only the energy efficiency and network management cost reduction but also solves the problem non-reliable nodes data dissemination. First we proposed a novel energy-efficient and distributed network management cost minimization framework for dynamic connectivity and data dissemination in opportunistic WBANs. Then proposed the pricing based approach for reliable node data dissemination.

We introduced a pricing based approach to optimize the network management cost for opportunistic WBANs. Concurrently, the behaviors of WBANs are taken into the consideration (i.e., critical and normal condition) to provide reliable services. We proposed algorithms: (1) energy efficient prioritized opportunistic communications algorithm, (2) optimal network cost reduction algorithm.

As the dynamic topological disconnections as well as variations in link qualities increases the energy consumption rate of opportunistic WBANs, we design the energy-efficient prioritized opportunistic communication to optimize the energy consumption rate of WBANs. Algorithm 1 shows the process for energy consumption minimization [19].

\section{Algorithm 1: Energy Efficient Algorithm}

\section{Inputs:}

- B: Number of WBANs.

- AP: Number of APs

- b: number of sensor nodes.

- T: Total time period

Output: Energy consumption rate $\left(\mathrm{F}_{\mathrm{ec}}^{\mathrm{t}^{*}}\right)$.

1: Measure $\mathrm{x}_{\mathrm{ij}}^{\mathrm{intra}}$ and $\mathrm{X}_{\mathrm{ij}}^{\mathrm{inter}}$ at time $\mathrm{t}$.

2: Calculate $\mathrm{f}_{\mathrm{ij}}(\mathrm{t})$ and $\mathrm{R}_{\mathrm{ij}}^{\mathrm{a}}(\mathrm{t})$ at time $\mathrm{t}$.

3: if $\left(\xi_{\text {decision }}^{t} \geq \xi_{\text {decision }}^{t}\right)$ then

4: Connectivity establishment

5: Calculate $\mathrm{F}_{\mathrm{ec}}^{\mathrm{t}}$ at time $\mathrm{t}$.

6: if $\left(\mathrm{F}_{\mathrm{ec}}^{\mathrm{t}} \geq \mathrm{F}_{\mathrm{ec}}^{\mathrm{th}}\right)$ then

7: Plan opportunistic energy limitation parameter.

8: Plan optimization issues through the Equation 24.

9: end if

10: if $\left(\mathcal{P}_{\mathrm{i}} \geq \mathcal{P}^{\text {th }}\right)$ then

11: Update waiting time $\mathrm{T}_{\text {wait }}=\mathrm{T}_{\text {low }}$.

12: Optimal energy utilization price $\left(\mathrm{F}_{\mathrm{ec}}^{\mathrm{t}^{*}}\right)$.

13: end if

14: end if

15: Update $\mathrm{T}_{\text {wait }}^{*}=\left(\mathrm{T}_{\text {low }}+1\right)$.

16: Return when $T^{*}=T_{\text {tot }}$. 


\section{International Journal of Innovative Research in Electrical, Electronics, Instrumentation and Control Engineering}

Vol. 6, Issue 11, November 2018

Where, $\mathrm{X}_{\mathrm{ij}}^{\mathrm{inter}}$ variable and $\mathrm{x}_{\mathrm{ij}}^{\mathrm{intra}}$ variable are representing that the inter-BAN and intra-BAN links among ith sensor node and jth LPU node. The decision matrices are $\xi_{\text {decision }}^{t}$ is executed to recognize the opportunistic connectivity for intra- BAN and inter-BAN transmission units, based on the connection quality and propagation delay at time $t$.

Further to decreases the network management costs, we investigate another set of rules for most efficient cost minimization algorithm. This set of rules consists of, interference control price, electricity management value, records distributed cost, and QoS-making sure cost. Algorithm 2 represents the technique to decrease the community management fee.

\section{Algorithm 2: Cost Minimization Algorithm}

\section{Inputs:}

- B: Number of WBANs.

- AP: Number of APs

- b: number of sensor nodes.

- T: Total time period

\section{Output: Cost Management Matrix $\left(\mathrm{CM}_{\mathrm{i}, \mathrm{j}}^{*}\right)$.}

1. Measure $\mathrm{X}_{\mathrm{ij}}^{\mathrm{intra}}$ and $\mathrm{X}_{\mathrm{ij}}^{\mathrm{inter}}$ at time t.

2. Calculate $C_{\mathrm{X}_{\mathrm{ij}}}^{\text {intra }}$ and $\mathrm{C}_{\mathrm{X}_{\mathrm{ij}}}^{\text {inter }}$ at time $\mathrm{t}$.

3. if $\xi_{\text {decision }}^{t} \geq \xi_{\text {decision }}^{t}$ then

4. Opportunistic connectivity extraction

5. Update waiting time $\mathrm{T}_{\text {wait }}=\mathrm{T}_{\text {low }}$

6. if $\mathrm{C}_{\mathrm{OC}}^{\mathrm{t}} \geq \mathrm{C}_{\mathrm{OC}}^{\text {th }}$ then

7. Calculate $\mathrm{C}_{\mathrm{DC}}^{\mathrm{t}} \mathrm{C}_{\text {tot }} \mathrm{C}_{\text {inff }}^{\mathrm{t}}$ tot .

8. Calculate $\mathrm{C}_{\text {qos tot }}^{\mathrm{t}}, \mathrm{C}_{\mathrm{E}_{\text {tot }}^{\mathrm{t}}}^{\mathrm{tot}}$

9. end if

10. if $\mathrm{C}_{\mathrm{tot}}^{\mathrm{t}} \geq \mathrm{C}_{\mathrm{tot}}^{\mathrm{th}}$ then

11. Optimize the network management cost

12. Optimal Network Management Cost Management Matrix $\left(\mathrm{CM}_{\mathrm{i}, \mathrm{j}}^{*}\right)$.

13. end if

14. end if

15. Update $\mathrm{T}_{\text {wait }}^{*}=\left(\mathrm{T}_{\text {low }}+1\right)$.

16. Return when $\mathrm{T}^{*}=\mathrm{T}_{\text {tot }}$.

Where, $C_{\mathrm{x}_{\mathrm{ij}}}^{\text {intra }}$ and $\mathrm{C}_{\mathrm{X}_{\mathrm{ij}}}^{\mathrm{inter}}$ are intra-BAN link cost and inter-BAN link cost respectively. $\mathrm{C}_{\mathrm{DC}}^{\mathrm{t}}$ tot and $\mathrm{C}_{\text {inff }}^{\mathrm{t}}$ tot are Data dissemination and interference management costs respectively. The $\mathrm{C}_{\mathrm{qos}}^{\mathrm{t}}$ tot and $\mathrm{C}_{\mathrm{E}_{\text {tot }}}^{\mathrm{t}}$ Are QoS ensuring cost and energy management costs respectively?

Lastly, to effective the dependable data communication model, we implemented the pricing method in this innovation paper. The pricing techniques recompense the nodes that communicate to another node packet and energy those that forward packet. The secure system analysis the nodes reliability and competence in a communicated packet in concepts of multi-dimensional trust values. They assumed values are connected to the hubs' open key authentication to be utilized as a part of making routing choices. We grow routing strategy to transmit the records through the ones observably relied upon hubs having the adequate energy to restrain the chance of breaking the route. This improved procedure can deal with route strength and the report precise battery energy ability.

This is because of the reality any loss of considering will bring about the loss of destiny income. Besides, for the proficient usage of the think about the packet, they trust values are registered by utilizing preparing the value receipts. . Figure 2: Pricing and incentive mechanism. 


\section{International Journal of Innovative Research in Electrical, Electronics, Instrumentation and Control Engineering}

Vol. 6, Issue 11, November 2018

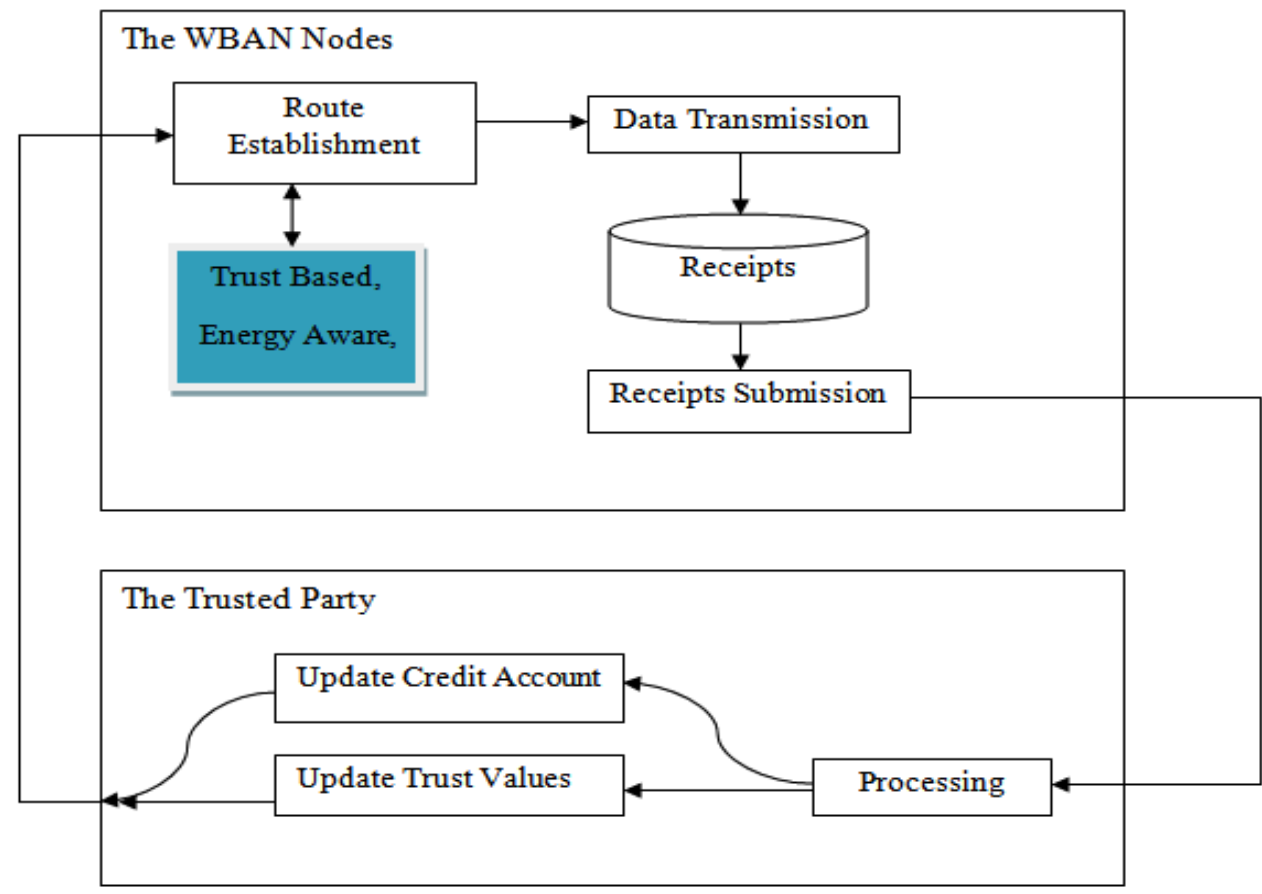

Figure 1: Pricing based data transmission

\section{SIMULATION RESULTS}

In this area, we display the present outcomes utilizing the network simulator NS2. We evaluated the methods using the ns-2.34 version. The network parameters and techniques represented in table 1. The proposed RNCDM method is compared with two state-of-art methods in this section such as OPS [19] and NCMD [19].

Table 1: Network Simulation Parameters

\begin{tabular}{|l|l|}
\hline Network Area & $1000 \times 1000$ \\
\hline Type of Network & WBAN \\
\hline Number of Nodes & $50-350$ \\
\hline Velocity & $1.5 \mathrm{~m} / \mathrm{s}$ \\
\hline MAC & 802.11 \\
\hline Simulation Time & $30 \mathrm{sec}$ \\
\hline Initial Energy & $0.5 \mathrm{~J}$ \\
\hline Transmitter energy consumption & $16.7 \mathrm{~nJ}$ \\
\hline Receiver energy consumption & $36.1 \mathrm{~nJ}$ \\
\hline
\end{tabular}

There are five performance metrics evaluated in this paper such as:

Energy Consumption vs. Number of WBANs

Data Dissemination delay vs. Number of WBANs

Network Throughput vs. Number of WBANs

PDR vs. Number of WBANs

Number of packet drops vs. Number of WBANs

Table 2: Average throughput performance evaluation

\begin{tabular}{|l|l|l|l|}
\hline WBANs & OPS & NCMD & RNCMD \\
\hline 50 & 250 & 260 & 270 \\
\hline 100 & 253 & 262 & 271 \\
\hline 150 & 261 & 275 & 293 \\
\hline 200 & 272 & 285 & 300 \\
\hline 250 & 288 & 303 & 321 \\
\hline 300 & 305 & 316 & 327 \\
\hline 350 & 311 & 327 & 342 \\
\hline
\end{tabular}




\section{International Journal of Innovative Research in Electrical, Electronics, Instrumentation and Control Engineering}

Vol. 6, Issue 11, November 2018

Table 3: PDR performance evaluation

\begin{tabular}{|l|l|l|l|}
\hline WBANs & OPS & NCMD & RNCMD \\
\hline 50 & 67.5 & 70 & 73 \\
\hline 100 & 68 & 71 & 74 \\
\hline 150 & 72.5 & 75 & 78 \\
\hline 200 & 76 & 79 & 82 \\
\hline 250 & 79 & 82 & 85 \\
\hline 300 & 83 & 86 & 88 \\
\hline 350 & 85 & 88 & 91 \\
\hline
\end{tabular}

As showing in the performance of throughput and PDR, the proposed RNCDM technique improves the performance due to the effective strategies designed for the optimum opportunistic routing. The RNCDM is based on NCDM technique.

Table 4: Average energy consumption performance evaluation

\begin{tabular}{|l|l|l|l|}
\hline WBANs & OPS & NCMD & RNCMD \\
\hline 50 & 0.072 & 0.079 & 0.095 \\
\hline 100 & 0.075 & 0.08 & 0.097 \\
\hline 150 & 0.081 & 0.09 & 0.102 \\
\hline 200 & 0.1 & 0.109 & 0.122 \\
\hline 250 & 0.12 & 0.129 & 0.142 \\
\hline 300 & 0.142 & 0.15 & 0.163 \\
\hline 350 & 0.163 & 0.17 & 0.19 \\
\hline
\end{tabular}

Table 5: Number of packets drop performance evaluation

\begin{tabular}{|l|l|l|l|}
\hline WBANs & OPS & NCMD & RNCMD \\
\hline 50 & 590 & 540 & 490 \\
\hline 100 & 575 & 525 & 475 \\
\hline 150 & 500 & 450 & 400 \\
\hline 200 & 425 & 375 & 325 \\
\hline 250 & 375 & 325 & 275 \\
\hline 300 & 310 & 260 & 210 \\
\hline 350 & 260 & 260 & 160 \\
\hline
\end{tabular}

Table 6: Data dissemination delay performance evaluation

\begin{tabular}{|l|l|l|l|}
\hline WBANs & OPS & NCMD & RNCMD \\
\hline 50 & 1.79 & 1.7 & 1.62 \\
\hline 100 & 1.35 & 1.3 & 1.23 \\
\hline 150 & 0.8 & 0.79 & 0.78 \\
\hline 200 & 0.48 & 0.46 & 0.44 \\
\hline 250 & 0.39 & 0.38 & 0.38 \\
\hline 300 & 0.28 & 0.25 & 0.22 \\
\hline 350 & 0.18 & 0.17 & 0.16 \\
\hline
\end{tabular}

Similarly, the delay and average energy consumption performance is optimized using the proposed solution for the varying WBANs. The throughput and PDR performances are increasing with increased number of WBANs.

\section{CONCLUSION AND FUTURE WORK}

In this research, we proposed the network management cost reduction approach for the opportunistic WBANs in order to manage the increased cost of network management. We first designed the joint distributed network management cost reduction algorithm and energy-efficient algorithm for the dynamic data dissemination process in the opportunistic WBANs. Then we introduced pricing based data transmission for the reliable and stable route selection. The simulation results prove that proposed solution outperforms the existing routing protocols for WBANs. For future work, it will be interesting to investigate the variations in other important parameters of WBAN such as mobility speed, packet rate etc. 


\section{International Journal of Innovative Research in Electrical, Electronics, Instrumentation and Control Engineering}

Vol. 6, Issue 11, November 2018

\section{REFERENCES}

[1]. Benoit Latre, Bart Braem, Ingrid Moerman, Chris Blondia \& Piet Demeester 2011, „A Survey on Wireless Body Area Networksee, Journal of Wireless Networks, vol. 17, no. 1, pp. 1-18.

[2]. Kyung Sup Kwak, Sana Ullah \& Niamat Ullah 2010, "An Overview of IEEE 802.15.6 Standarde, 3rd International Symposium on Applied Sciences in Biomedical and Communication Technologies (ISABEL), 7-10 Nov. 2010 Rome, Italy, pp. 1-6.

[3]. M. Chen, S. Gonzalez, A. Vasilakos, H. Cao, and V. C. Leung, "Body Area Networks: A Survey," Journal of Mobile Networks and Applications, vol. 16, no. 2, pp. 171-193, 2011.

[4]. S. Moulik, S. Misra, and A. Gaurav, "Cost-Effective Mapping Between Wireless Body Area Networks and Cloud Service Providers Based on Multi-Stage Bargaining,” IEEE Transactions on Mobile Computing, vol. PP, no. 99, pp. 1-1, 2016.

[5]. K. M. S. Thotahewa, J. Y. Khan, and M. R. Yuce, "Power Efficient Ultra Wide Band Based Wireless Body Area Networks with Narrowband Feedback Path," IEEE Transactions on Mobile Computing, vol. 13, no. 8, pp. 1829-1842, 2014.

[6]. S. Ullah, H. Higgins, B. Braem, B. Latre, C. Blondia, I. Moerman, S. Saleem, Z. Rahman, and K. S. Kwak, "A Comprehensive Survey of Wireless Body Area Networks," Journal of Medical Systems, vol. 36, no. 3, pp. 1065-1094, 2012.

[7]. "IEEE Standard for Local and Metropolitan Area Networks - Part 15.6: Wireless Body Area Networks,” IEEE Std 802.15.6-2012, pp. 1-271, 2012

[8]. A. Samanta, S. Bera, and S. Misra, "Link-Quality-Aware Resource Allocation With Load Balance in Wireless Body Area Networks," IEEE Systems Journal (DOI: 10.1109/JSYST.2015.2458586), vol. PP, no. 99, pp. 1-8, 2015.

[9]. A. Samanta, S. Misra, and M. S. Obaidat, "Wireless Body Area Networks with Varying Traffic in Epidemic Medical Emergency Situation," in Proceedings of IEEE International Conference on Commumications, 2015.

[10]. J. Elias, "Optimal Design of Energy-efficient and Cost-effective Wireless Body Area Networks,” Ad Hoc Networks (Elsevier), vol. 13, pp. 560$574,2014$.

[11]. M. Zhao, D. Gong, and Y. Yang, "Network Cost Minimization for Mobile Data Gathering in Wireless Sensor Networks," IEEE Transactions on Communications, vol. PP, no. 99, pp. 1-1, 2015.

[12]. S. Yousaf, N. Javaid, Z. A. Khan, U. Qasim, M. Imran, and M. Iftikhar, "Incremental Relay Based Cooperative Communication in Wireless Body Area Networks,” Procedia Computer Science, vol. 52, pp. 552-559, 2015.

[13]. F. D. Andreagiovanni and A. Nardin, "Towards the Fast and Robust Optimal Design of Wireless Body Area Networks," Applied Soft Computing, vol. 37, pp. 971-982, 2015.

[14]. S. Huang and J. Cai, "Priority-Aware Scheduling for Coexisting Wireless Body Area Networks," in Proceedings of International Conference on Wireless Communications Signal Processing, 2015, pp. 1-5.

[15]. E. Ibarra, A. Antonopoulos, E. Kartsakli, J. Rodrigues, and C. Verikoukis, "QoS-Aware Energy Management in Body Sensor Nodes Powered by Human Energy Harvesting," IEEE Sensors Journal, vol. 16, no. 2, pp. 542-549, 2016.

[16]. A. Seyedi and B. Sikdar, "Energy Efficient Transmission Strategies for Body Sensor Networks with Energy Harvesting," IEEE Transactions on Communications, vol. 58, no. 7, pp. 2116-2126, 2010.

[17]. "Modeling and Analysis of Energy Harvesting Nodes in Body Sensor Networks," in Proceedings of International Summer School and Symposium on Medical Devices and Biosensors, 2008, pp. 175-178.

[18]. Z. Ren, X. Qi, G. Zhou, H. Wang, and D. Nguyen, "Throughput Assurance for Multiple Body Sensor Networks," IEEE Transactions on Parallel and Distributed Systems, vol. PP, no. 99, pp. 1-1, 2015

[19]. Amit Samanta, Student Member, IEEE, Sudip Misra, "Energy-Efficient and Distributed Network Management Cost Minimization in Opportunistic Wireless Body Area Networks", IEEE Transactions on Mobile Computing, 2017 